\title{
UNE MÉTHODE PRATIQUE D'EXÉCUTION DES CALCULS GRAPHIQUES
}

\author{
Y. PONSAR \\ Ingénieur I. C.A.M.
}

\section{I. - INTRODUCTION}

Les calculs graphiques sont d'un emploi très courant en hydraulique. Dans les intégrations, ils permettent d'aller plus loin que l'analyse ne saurait le faire : pour l'étude de certains phénomènes d'allure discontinue, scomme le sont les coups de bélier et le's intumescences, les calculs graphiques semblent même être les seuls praticables.

C'est probablement dans le domaine des coups de bélier que ces méthodes s'imposent avec le plus d'évidence. Sans rien vouloir enlever aux mérites d'Alrı́vi dans l'étude de's surpressions, il faut reconnaître que l'ingénieur moyen a dù attendre lapparition de la méthode graphique pour comprendre véritablement la question, et, qu'à ce moment, il a su d'un seul coup traiter facilement la plupart dés problèmes et suivre très clairement le jeu des ondes, jeu mystérieux et déroutant au premier abord.

L'ingénieur le plus sérieux fut conquis peu à peu par ces jeux : il prit part aux courses de relais que les observateurs de M. L. Bergeron font sans cesse, a la partie de cache-cache à laquelle ils se livrent dan's les branchements, à leur jeu de balle au mur sur quelque obturateur fe'rmé.

Mais prenez garde, ces courses folles ne sont pas sans danger, ne vous grisez pas de vitesse, un faux pas risque de tout brouiller.

C'est pour éviler ces faux pas que nous avons cherché à simplifier le tracé des épures et nous pensons y etre parvenus, puisque, dans bien des cas, le tracé de toute ligne est supprimé, le travail graphique se réduisant à pointer et numéroter les points caractéristiques représentant la surpression et le débit à chaque intervalle de temps.

\section{II. -- METHODE HABITUELLE}

Pour mieux nous fare comprendre, nous nous voyons dans l'obligation de donner un exemple en nous excusant du surcroît de travail que nous imposons au lecteur. Choisissons cet exemple très simple en considérant une installation composée d'un bassin à niveau invariable, d'une. conduite sans pertes de charge, d'une chemince d'équilibre simple sans diaphragme, d'un obtarateur fixé en position et débitant à l'air libre.

Nous supposerons que l'installation est en cours d'oscillations et étudierons ce mouvement. qui est en masse.

Représentons par le point $o b$ dans le plan $\mathrm{QH}$ habituel, un état quelconque « pressiondébit $\gg$ en $b$, au temps 0 (zéro) qui est pris comme origine des temps.

Nous pouvons, sur l'épure, tracer la droite Bergeron $o b-1 a$ sur laquelle se trouvent les états rencontrés par l'observateur remontant au réservoir.

Le point $1 a$ est l'état «pression-débit 》 en $a$, au temps 1 (unité de temps) $=\frac{\mathrm{L}}{a}$ avec $\mathrm{L}=$ longueur de la conduite et $a=$ célérité des ondes, pente de la droite $\frac{a}{g s}$ avec $s=$ section de la conduite; la droite $1 a-2 b$ représente ce que voit ensuite cet observateur redescendant la conduite depuis le réservoir et arrivant au temps 2 à l'extrémité aval.

Nous pouvons, d'autre part, évaluer la montée du niveau dans la cheminée pendant les intervalles de temps $0-1$ et 1-2 par les droites $o b-1$ et $1-2 b$ construites sur une distance horizontale qui représente la différence entre les débits de la conduite et de l'obturateur, c'est-àdire le débit entrant dans la cheminée (la pente de ces droites est $\pm \frac{\Delta t}{\mathrm{~S}}= \pm \frac{\mathrm{L}}{a s}$ avec $\mathrm{S}$ section de la cheminée et $\Delta t=\frac{\mathrm{I}}{a}$ ).

Pour ne rien compliquer, nous nous tiendrons à cette construction simplifiée.

Le point $2 b$ se trouve donc détérminé par l'intersection d'une droite BERgEron et d'une droite de montée d'eau. On peut recommencer 


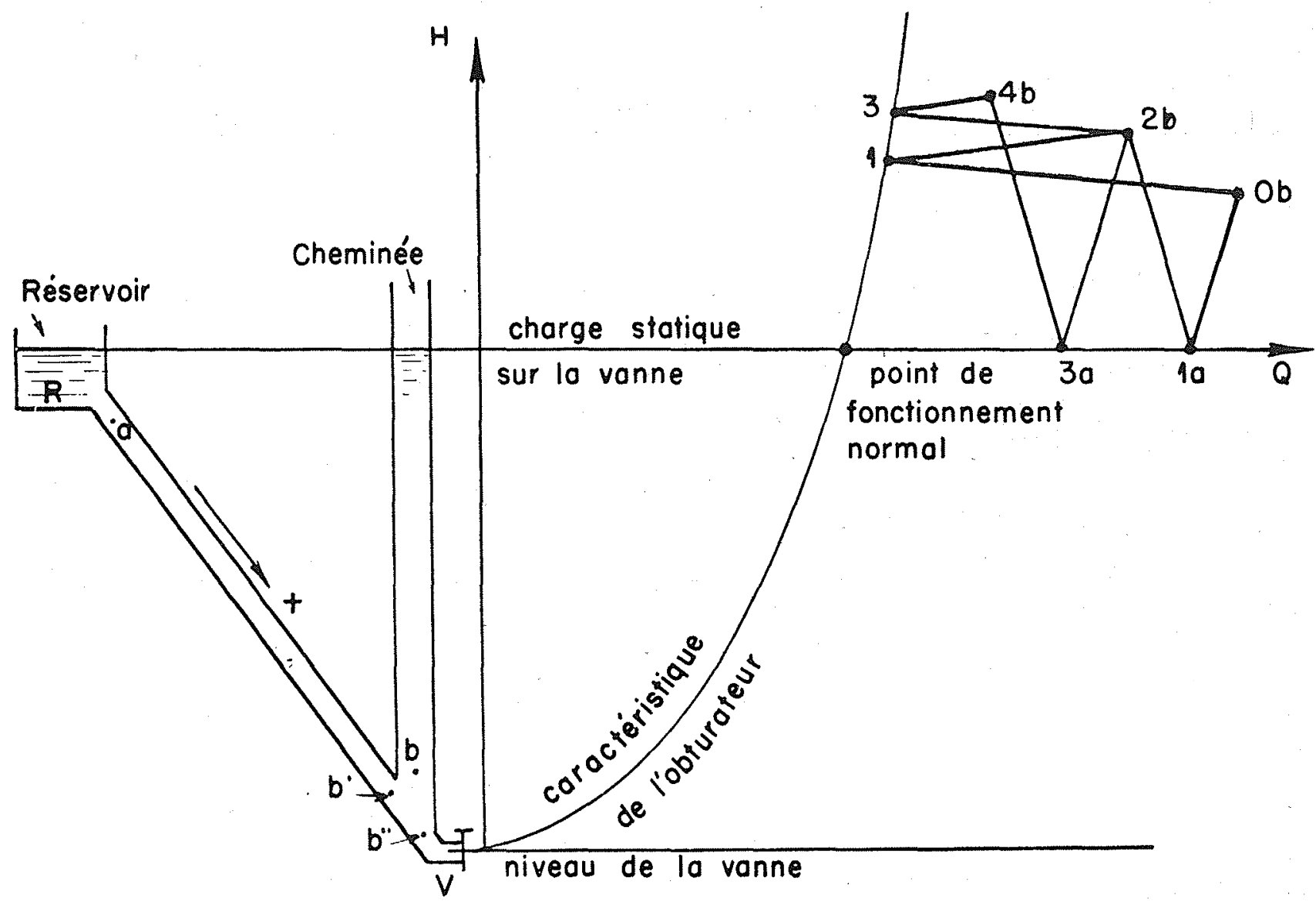

la même construction pou'r trouver le point $3 b$ et ainsi de suite.

\section{III. - METHODE ACCELEREE}

Nous constatons sur l'épure (fig. 1), qu'en chaque point $b$ convergent quatre droites de pentes déterminées, ce qui conduit à la méthode pratique de tracé suivante, qui ne changeant rien au principe ci-dessus, e'st simplement plus rapide.

Sur un papier blanc fixe, appelé canevas, nous tracerons à partir d'un point, le faisceau de deux droites de pente $\pm \frac{a}{g s}$ et le faisceau de deux droites de pente $\pm \frac{\mathrm{L}}{a} \mathrm{~S}^{-}$

L'épure se construira sur un papier-calque, que nous déplacerons parallèlement à lui-même sur le papier blanc. Connaissant les points $1 a$ et 1 pointés en premier lieu sur le calque, nous les ferons coïncider par transparence, le premier avec la droite Bergeron de pente $-\frac{a}{g}-$, le second avec la montée de pente $+\frac{\mathrm{L}}{a \mathrm{~S}}$.

L'emplacement de $2 b$ sera pointé sur l'épure et, en même tempss, on pointera les emplacements de $3 a$ et 3 .

A partir de ces deux derniers points, la construction sera répétée pour obtenir en nême temps, $4 b, 5 a$ et 5 et ainsi de suite.

\section{IV. - AUTRES PROBLEMES}

Nous avon's pris intentionnellement un exemple très simple; pour des cas plus compliqués, on devra chercher par des artifices de tracé, à se rapprocher du schéma précédent; l'ingéniosité du dessinateur pourra se donner libre cours. La méthode consistera souvent à tracer sur ur papier millimétré, fixé sur une planche à dessin, les différentes droites et courbes dont on a besoin d'une façon répéiée ; pour tracer l'épure (droites d'onde, droites de montée d'eau dans les bassins, parabole de pertes de charge, hyperbole représentant la loi de compression de poches ou de réservoirs d'air ou celle représentant une turbine munie de son régulateur) ces courbes 
donneront le plus souvent naissance à des courbes conjuguées par combinaisons de plusieurs d'entre elles.

Un caneva's étant ainsi tracé et fixé sur une planche à dessin, l'épure sera construite sur le papier-calque fixé lui-même à un cadre pouvant se déplacer parallèlement à lui-même.

Le plu's pratique est de remplacer l'équerre habituelle d'un appareil à dessiner du commerce par un cadre comme le représente la figure cidessous.

La construction la pilus simple ver's laquelle on doit tendre consiste à tout réduire, sur le caneva's, à quatre courbes analogues aux quatre droites de l'exemple précédent.

Le travail se poursuivra sans interruption à la pointe $d u$ crayon sans maniement d'aucune règle ou équerre. Une telle méthode extrême- ment rapide peut être trouvée pour l'étude des petites oscillations de l'installation choisie dans l'exemple en lui ajoutant une turbine munie d'un régulateur réglant la puissance constante, un diaphragme à la base de la cheminée, des pertes de charge dans la conduite. Un tel en'semble est déjà assez complexe, et il est curieux de constater que son étude pent se ramener au maniement simple de 6 courbes ou droites fixes (4 sur le canevas, 2 sur le calque).

La nature n'a pas toujours une telle bonne volonté et l'on ne s'étonnera pas que dans d'autres cas, la construction se complique, légèrement d'ailleurs, et que l'on soit conduit au report de certains points pris sur une nouvelle courbe.

L'exposé d'exemples sur ces autres cas sortirait du cadre de cette note et nous laisserons, aujourd'hui, le lecteur travailler seul dans ce

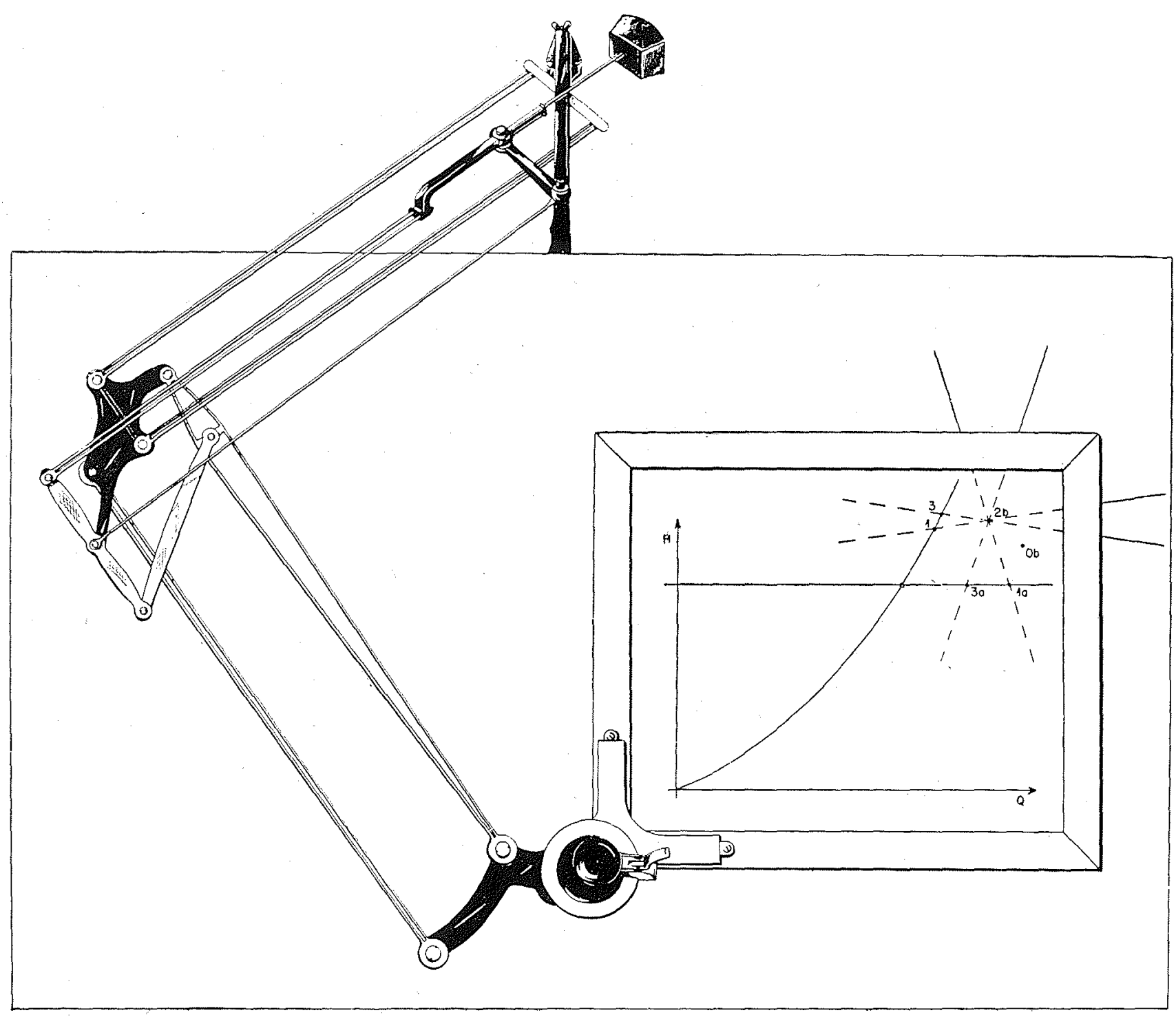


sens ; dès qu'il sera familiarisé avec son appareil, il abordera des problèmes véritablement compliqués et sera surpris de constater qu'il opère beaucoup plus vite, avec une précision au moins aussi bonne qu'avec les moyens habituels.

\section{V. - EXTENSION DE LA METHODE}

Il est évident que le très vaste domaine des coups de bélier n'est qu'un des champs d'application de la méthode. Elle peut ètre transposée pour l'étude de tous les phénomènes vibratoires mentionnés par M. L. Brergeron et, en particulier, aux vibrations transversales de cordes tendues, aux vibration's de torsion ou de compression de barres ou de ressorts, aux oscillations électriques et généralement à toutes propagations d'ondes planes.

La rapidité du tracé rend possible pratiquement l'étude graphique d'oscillations en masse, beaucoup plus longues et majestueuses que les. coups de bélier d'onde et dont l'étude serait pour le moins lassante, si tous les traits devaient être tracés.

Nous l'avons appliquée aussi à certaines études de régulation et parliculièrement à la régulation de vamnes automatiques où jouaient l'inertie de la vanne, la surface de divers bassins, des dispositifs stabilisateur's. Il serait peut-ètre intéressant de rapprocher de ces problèmes de régulation, les études de phénomènes cycliques de tous ordres.

Un autre domaine dans l'hydraulique paraît devoir être celui des courbes de remous dans les canaux prismatiques.

\section{VI. - CONCLUSION}

Des problèmes cités ci-dessus, certains sont pratiquement inabordables au calcul analytique ; d'autres le sont, mais au prix de certaines hypothèses simplificatrices ; d'autres enfin sont accessibles au calcul pur, et ceci en toute rigueur.

Les méthodes de calculs graphiques s'imposent pour la première catégorie, elles peuvent être utiles pour la seconde en permettant d'obtenir une meilleure précision dans les résultats, enfin, pour la dernière, elles ne sauraient être intéressantes que si elles conduisent à une vue plus claire des phénomènes (ce qui peut être une question de tournure d'esprit) ou à un gain de temps appréciable.

L'emploi des panneaux mobiles transparents (dont M. d'OC.agne faisait déjà un large usage dans ses abaques, en se servant de plusieurs calques superposés) supprim l'obligation matérielle de tracer la plupart cies lignes de construction; cet emploi accélère très sensiblement le tracé et peut donc faire pencher la balance, mème pour certains cas de la dernière catégorie en faveur de la méthode graphique. 